\title{
Inserção dos Cuidados Paliativos na formação dos profissionais de educação física
}

\section{Insertion of Palliative Care in the training of physical education professionals}

\section{AUTORES \\ Breno Augusto Bormann de Souza Filho ${ }^{1}$ (D) Érika Fernandes Tritany ${ }^{2}$ (D) \\ William Serrano Smethurst ${ }^{3}$ (i) \\ Mauro Virgilio Gomes de Barros ${ }^{3}$ (D) \\ 1 Escola Nacional de Saúde Pública, Departamento de Epidemiologia, Rio de Janeiro, Rio de Janeiro, Brasil \\ 2 Universidade Federal do Rio de Janeiro, Instituto de Estudos em Saúde Coletiva, Rio de Janeiro, Rio de Janeiro, Brasil. \\ 3 Universidade de Pernambuco, Departamento de Educação Física, Recife, Pernambuco, Brasil.}

\section{CONTATO}

Breno Augusto Bormann de Souza Filho brenobormann@botmail.com

Avenida Prado Júnior. n. 330, Copacabana, Rio de Janeiro, Rio de Janeiro, Brasil. CEP: 22011-040.

DOI

$10.12820 /$ rbafs.26e 0184

\section{(cc) BY}

Este trabalho está licenciado com uma Licença Creative Commons - Atribuição 4.0 Internacional.

\begin{abstract}
RESUMO
A recente Resolução no 391 do Conselho Federal de Educação Física, de agosto de 2020, estimulou o debate acerca da ampliação do horizonte de atuação do Profissional de Educação Física (PEF) no Brasil, ao definir sua atuação em contextos hospitalares, em atividades relacionadas às áreas de atividades físicas e exercício físico, destinados a promoção, prevenção, proteção, educação, intervenção, recuperação, reabilitação, tratamento e Cuidados Paliativos. Nesse sentido, é importante que os PEF adquiram, ao longo de sua formação, conhecimentos e práticas relacionados aos Cuidados Paliativos (CP). Entretanto, são observadas lacunas na formação profissional e ausência de orientações na Diretriz Curricular Nacional sobre competências, habilidades e atitudes, no que tange a cuidados durante o percurso das doenças, cuidados de fim de vida, luto, e aspectos conceituais dos $\mathrm{CP}$ na formação básica do PEF. Assim, este artigo discute a importância da atuação do PEF nos $\mathrm{CP}$, bem como a inclusão de conteúdos próprios à área dos $\mathrm{CP}$ em componentes curriculares da formação em Educação Física. Propõe-se, assim, ampliar e qualificar a atuação do PEF, e melhorar a qualidade da assistência prestada a pacientes, e seus familiares, em CP.
\end{abstract}

Palavras-chave: Cuidados paliativos; Capacitação de recursos humanos em saúde; Capacitação professional; Currículo.

\section{ABSTRACT}

The recent Resolution $n^{\circ} 391$ of the Federal Council of Physical Education, stimulated the debate about the expansion of the horizon of action of the Professional of Physical Education (PPE) in Brazil, when defining his performance in hospital contexts, in activities related to the areas physical activities and physical exercise, aimed at promotion, prevention, protection, education, intervention, recovery, rehabilitation, treatment and Palliative Care (PC). In this sense, it is important that the PEF acquire, throughout their training, knowledge and practices related to Palliative Care (CP). However, gaps in professional training are observed and there is no guidance in the National Curricular Guideline on competences, skills and attitudes, regarding care during the course of illnesses, end of life care, mourning, and conceptual aspects of $P C$ in basic training of the PEF. Thus, this article discusses the importance of the performance of the PPE in the PCs, as well as the inclusion of content specific to the PCs area in the curricular components of Physical Education training. Thus, it is proposed to expand and qualify the performance of the PPE, and to improve the quality of care provided to patients, and their families, in Palliative Care.

Keywords: Palliative care; Health human resource training; Professional training; Curriculum.

\section{Introdução}

Cuidado Paliativo $(\mathrm{CP})$ é uma abordagem que visa a prevenção e alívio do sofrimento, promoção de dignidade, melhoria da qualidade de vida e adaptação a doenças e/ou condições progressivas em pessoas que enfrentam problemas de saúde crônicos, complexos ou limitadores da vida e para suas famílias. Dessa forma, requer identificação precoce, avaliação e tratamento de sintomas de natureza física, psicossocial e espiritual ${ }^{1,2}$.

Nos CP, a preservação da funcionalidade global dos pacientes e de seus familiares/cuidadores durante todo o processo de apoio e cuidado é essencial ${ }^{3}$. Assim, es- timula-se que os serviços de saúde adotem estratégias de reabilitação focadas na manutenção e promoção da independência funcional, prevenção e retardo de complicações deletérias ${ }^{1}$.

Os Cuidados Paliativos de Reabilitação integram, ao modelo holístico de CP, reabilitação, habilitação, autogestão e autocuidado. Trata-se de uma abordagem interdisciplinar, onde todos os profissionais envolvidos trabalham colaborativamente com o paciente e seus familiares/cuidadores para apoiá-los a alcançar seus objetivos e prioridades, com intuito de otimizar a função e o bem-estar, dentro das limitações do avanço 
da doença e/ou condição clínica vivenciada ${ }^{3}$. Objetiva-se melhorar a funcionalidade, dentro das capacidades de cada indivíduo; manter a função quando os efeitos da doença e/ou do tratamento ameaçam seu declínio; ou, ainda facilitar a transição para o declínio funcional quando a deterioração é inevitável ${ }^{3}$.

A Educação Física é uma área de conhecimento e de intervenção profissional que tem como objeto de estudo e aplicação a motricidade ou movimento humano, e a cultura do movimento corporal, com foco nas diferentes formas e modalidades do exercício físico, da ginástica, do jogo, do esporte, das lutas e da dança. Tem por objetivo atender às necessidades do campo da saúde, da educação e formação, da cultura, do alto rendimento esportivo e do lazer.

No ano de 2020, os Profissionais de Educação Física (PEF) foram reconhecidos na Classificação Brasileira de Ocupações (CBO) sob uma nova nomenclatura, "Profissional de Educação Física na Saúde”. Essa atualização da $\mathrm{CBO}$ representa um importante marco para essa categoria profissional, uma vez que possibilita que os PEF integrem formalmente as equipes multidisciplinares de saúde no âmbito dos serviços públicos e privados de saúde, o que favorece a contratação desses profissionais; define e regulamenta atividades de competências do PEF no setor saúde; e fortalece sua importância e participação nas ações e serviços no âmbito do Sistema Único de Saúde (SUS).

Adicionalmente, a Resolução no 391 do Conselho Federal de Educação Física, de 26 de agosto de $2020^{4}$, define a atuação do PEF nos contextos hospitalares, estabelecendo como prerrogativa deste profissional a coordenação, planejamento, programação, supervisão, dinamização, direção, organização, avaliação e execução de trabalhos, planos e projetos, nas áreas de atividades físicas e do exercício físico, destinados a promoção, prevenção, proteção, educação, intervenção, recuperação, reabilitação, tratamento e Cuidados Paliativos da saúde física e mental.

Entretanto, apesar da existência de prerrogativa legal para atuação do PEF no setor saúde e da compreensão acerca da importância deste profissional na composição de equipes multidisciplinares de saúde, nenhuma Diretriz Curricular Nacional (DCN) para os cursos de graduação em Educação Física, até o momento, apresenta as competências, habilidades e atitudes necessárias à formação do PEF para atuar de maneira oportuna nos Cuidados Paliativos. Portanto, é importante que tais considerações sejam incluídas de forma explícita nas DCNs, para orientação das Instituições de Ensino Superior (IES) na disposição de componentes curriculares que aproximem o estudante do ambiente profissional nos Cuidados Paliativos.

Nesse sentido, por se tratar de contexto de intervenção novo para profissionais da área de Educação Física, faz-se necessária discussão acerca da importância da inserção de componentes curriculares que abarquem as especificidades desse profissional em cada área de atuação. Assim, procurou-se neste texto discutir a importância da inclusão de conteúdos relacionados aos Cuidados Paliativos na formação inicial e continuada em Educação Física. Adicionalmente, apresentamos uma breve análise histórica do conceito de $\mathrm{CP}$ e seu desenvolvimento no Brasil, bem como seu modelo teórico de intervenção e competências necessárias para efetiva intervenção em CP.

\section{Um breve histórico conceitual sobre Cuidados Paliativos}

O conceito de CP foi introduzido em meados de 1960, na Inglaterra, por Cicely Saunders, apresentando uma filosofia de cuidado à pessoa com diagnóstico de doença incurável e em processo de terminalidade ${ }^{5}$. Ao longo dos anos, o conceito e abordagem dos $\mathrm{CP}$ passaram por intensas transformações ${ }^{6-8}$.

Frente à realidade mundial de transformações demográficas e epidemiológicas, com grande expressão das condições crônicas no perfil de morbimortalidade, foi divulgado pela Organização Mundial de Saúde (OMS), em 2017, um novo conceito que emerge como reorganizador das práticas de cuidado em saúde. Os $\mathrm{CP}$ passam a ser vistos como uma abordagem transversal a todas as etapas do cuidado, não apenas vinculados à terminalidade ou condições limitadoras da vida, como abordado no conceito formulado em 2002 pela OMS, mas também a todas as doenças crônicas progressivas. Exemplos comuns são pacientes com multimorbidade, fragilidade e demência; aqueles com falhas específicas de órgãos; ou acometidos por câncer, HIV/AIDS, malária, tuberculose resistente a medicamentos, diabetes, dentre outras ${ }^{1}$.

Pela definição atual, é reiterada a necessidade de consideração dos $\mathrm{CP}$ como direito humano básico e componente essencial de cuidados abrangentes e integrados ao longo da vida, inclusive no fim da vida e no acompanhamento do luto. Deve ser praticado por todos os prestadores de cuidados de saúde, compondo equipes multiprofissionais e iniciado o mais precocemente 
possível, concomitante ao tratamento modificador da doença ${ }^{1}$. Além disso, os $\mathrm{CP}$ apresentam como premissa do trabalho em saúde uma concepção de cuidado que sustente uma compreensão multidimensional de saúde; com especial atenção às dimensões física, emocional, social e espiritual da dor e sofrimento; não restrito apenas ao paciente, mas que se estenda a todos os envolvidos nesse processo - paciente/familiares/cuidadores e equipe multiprofissional ${ }^{1}$.

Desde o diagnóstico até o óbito, o processo de progressão da doença ou condição ameaçadora da vida, muitas vezes, é responsável por alterações na funcionalidade global do paciente, impactando negativamente na realização das Atividades de Vida Diária (AVDs), Atividades Instrumentais de Vida Diária (AIVDs) e, consequentemente, as Atividades Avançadas de Vida Diária (AAVD), o que pode incidir no aumento da sobrecarga dos familiares, cuidadores, e/ou profissionais envolvidos no cuidado ${ }^{9,10}$.

Nesse sentido, é importante que a abordagem paliativa se inicie precocemente, tão logo o problema de saúde seja detectado, atuando concomitantemente a possíveis tratamentos modificadores da doença ou condição - sejam eles relacionados a propostas curativas, para prolongar a vida, retardar a progressão da doença ou com intenção paliativa.

É importante ressaltar que, a depender da doença ou condição, os tratamentos específicos podem apresentar impacto limitado na modificação do curso da doença, prognóstico e qualidade de vida da pessoa em tratamento. Nesse sentido, é enfatizada a importância do aumento gradativo dos $\mathrm{CP}$, visando maior qualidade de vida ao paciente e sua família ${ }^{1}$, conforme modelo teórico apresentado na Figura 1.

\section{Cuidados Paliativos no Brasil}

A filosofia e abordagem dos CP ingressou no Brasil, na década de 1980 e, desde então, o país tem registrado crescente oferta de serviços focados no atendimento integral de pacientes e familiares, seguindo a definição e recomendações da $\mathrm{OMS}^{11}$. Nesse sentido, em 2018 foi publicada a Resolução no 41 , da Comissão Intergestores Tripartite ${ }^{12}$, a qual dispõe sobre as diretrizes para organização dos Cuidados Paliativos, à luz dos Cuidados Continuados Integrados, no Sistema Único de Saúde (SUS). Essa resolução materializa e dá institucionalidade a uma luta histórica pelo desenvolvimento e implementação dos $\mathrm{CP}$ no Brasil e sua inserção no SUS. A partir dela, fica acordado, nas três esferas de governo, a integração dos $\mathrm{CP}$ na Rede de Atenção

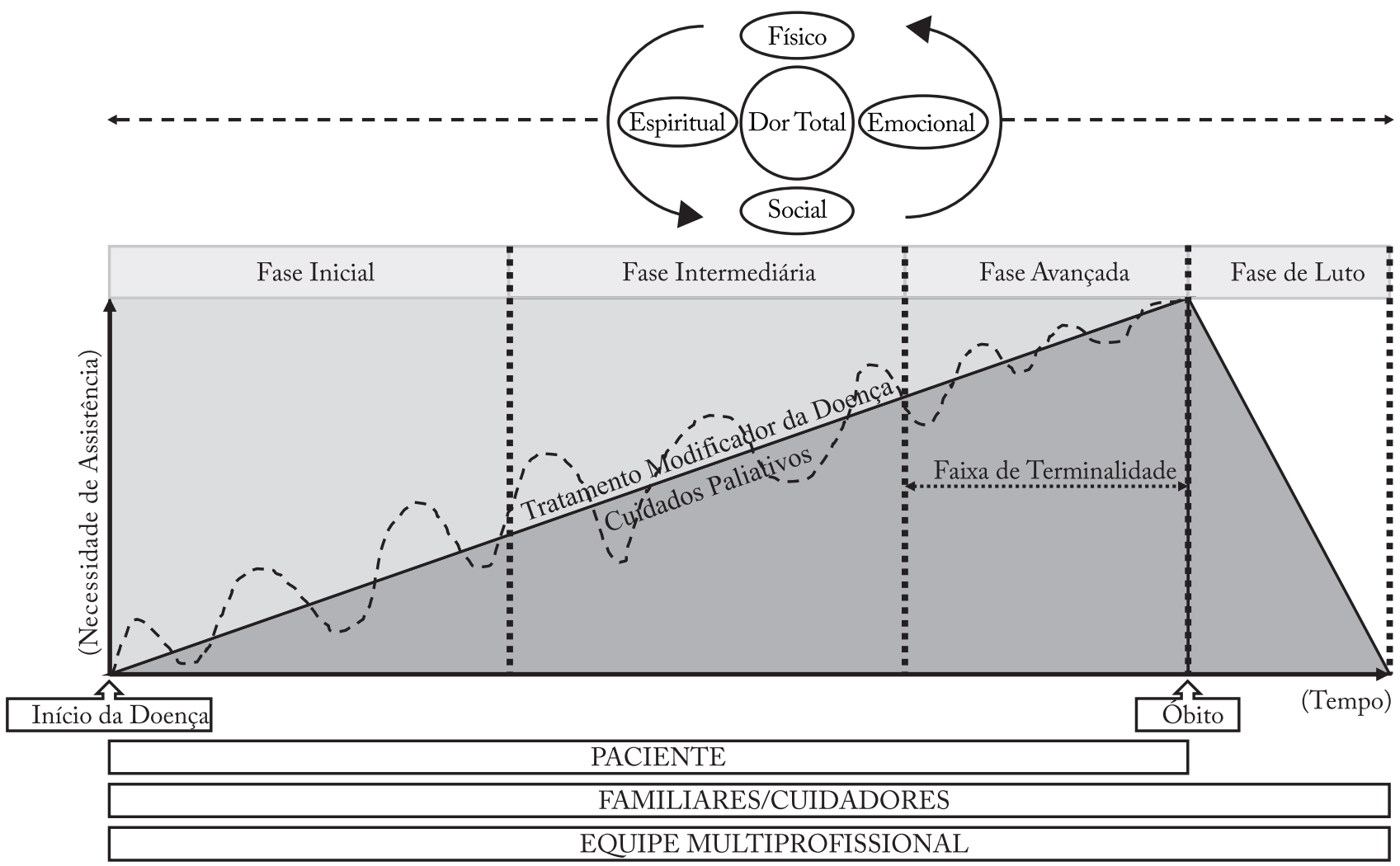

Figura 1 - Modelo Teórico para Implementação dos Cuidados Paliativos.

Fonte: elaboração própria, adaptado de Gómez-Batiste \& Connor ${ }^{1}$. 
à Saúde, sendo ofertados em qualquer ponto da Rede e coordenados pela Atenção Primária à Saúde (APS), com retaguarda nos demais níveis assistenciais. Além disso, é enfatizado o necessário fomento à inclusão de conteúdos programáticos sobre $\mathrm{CP}$ no ensino de graduação e pós graduação dos profissionais de saúde, bem como a oferta de educação permanente para os trabalhadores da saúde no SUS e a disseminação de informações sobre os Cuidados Paliativos na sociedade ${ }^{12}$.

Apesar disso, os desafios para a real implementação e disseminação dos CP no Brasil - e sobretudo no SUS - são inúmeros. Além da lacuna existente nas instituições de ensino e pesquisa na incorporação de conteúdos relacionados à $\mathrm{CP}$ no currículo básico de todas as graduações e pós-graduações da área da saúde ${ }^{1,13,14}$ no desenvolvimento de pesquisas na área e na formação de trabalhadores da saúde no SUS; ainda é forte na sociedade, nos serviços de saúde e no imaginário dos trabalhadores da saúde uma concepção incompleta e desatualizada sobre os $\mathrm{CP}$, associando-os e indicando-os apenas a pacientes em processo de fim de vida, e estereotipando o termo a uma noção errônea de "não ter mais o que se fazer" com o paciente.

Outrossim, a materialidade das ações preconizadas pela Resolução no 41 depende de uma série de fatores relacionados à priorização de ações e estratégias, pactuação integrada entre os entes federados, disponibilização de recursos, e do avanço de agendas políticas de enfrentamento aos desafios vivenciados pela gestão do sistema de saúde, fortalecimento das políticas públicas e do SUS.

\section{Competências centrais em CP necessárias para a formação profissional em saúde}

A Associação Europeia de Cuidados Paliativos (AECP) desenvolveu um guia ${ }^{15}$ que apresenta constituintes centrais que enquadram a aplicação dos princípios dos $\mathrm{CP}$ e reflete os valores subjacentes às melhores práticas, sob os tópicos: autonomia; dignidade; relação entre doente e profissionais de cuidados de saúde; qualidade de vida; posição em relação à vida e à morte; comunicação; educação pública; abordagem multiprofissional; perda e luto.

Além disso, o documento da AECP identifica como necessária a inclusão, nas diretrizes curriculares dos cursos de graduação em saúde, de conteúdos que fortaleçam dez competências centrais relacionadas aos $\mathrm{CP}$, quais sejam: 1) aplicar os constituintes centrais dos $\mathrm{CP}$ no ambiente próprio e mais seguro para pacientes e suas famílias; 2) aumentar o conforto físico durante as trajetórias de doença; 3) estar atento às necessidades psicológicas dos indivíduos em tratamento; 4) estar atento às necessidades sociais dos indivíduos; 5) estar atento às necessidades espirituais dos indivíduos; 6) responder às necessidades dos cuidadores familiares em relação aos objetivos do cuidar a curto, médio e longo prazo; 7) responder aos desafios da tomada de decisão clínica e ética em Cuidados Paliativos; 8) implementar uma coordenação integral do cuidado e trabalho em equipe interdisciplinar em todos os contextos de oferta de CP; 9) desenvolver competências interpessoais e comunicacionais adequadas aos $\mathrm{CP}$; e 10) promover o autoconhecimento e contínuo desenvolvimento profissional. Entretanto, tais competências ainda são pouco aprofundadas nos cursos de graduação atualmente ${ }^{15}$.

$\mathrm{O}$ guia da AECP propõe que essas competências sejam consideradas diretamente relevantes para a prestação da prática clínica de alta qualidade por qualquer profissional de saúde. Além disso, elas oferecem uma estrutura para o desenvolvimento de programas de educação em $\mathrm{CP}$, e uma base comum para apresentar o que é essencial para uma boa formação em CP. No entanto, é importante que tais competências, apresentadas pela EAPC, não sejam vistas meramente como uma ferramenta de desenvolvimento curricular per se. É importante o estabelecimento de um diálogo aberto, que permita um aprendizado transdisciplinar e que considere a perspectiva e vivência do outro. As competências supracitadas são globalmente relevantes para todos os que praticam $\mathrm{CP}$, independentemente da sua formação profissional, uma vez que são consideradas transferíveis para todos os cuidados necessários às pessoas com doenças e/ou condições que limitam a vida.

\section{Atuação e formação dos PEF e os Cuidados Paliativos}

As transições demográficas e epidemiológicas apresentam um cenário de emergência e preponderância de condições crônicas no perfil de morbimortalidade da população, o que requer uma abordagem complexa, coordenação e continuidade do cuidado e um sistema de saúde integrado, organizado sob Redes de Atenção à Saúde. Nesse contexto, ganha ênfase um cuidado em saúde pautado na tríade "prevenção, promoção e recuperação da saúde”.

No que tange a atuação do Profissional de Educação Física, evidencia-se - e é reforçado pela recente publicação da Resolução no 391 de 26 de agosto de 
$2020^{4}$ - seu papel fundamental na composição de equipes multiprofissionais de saúde, as quais se depararam, cada vez mais, com a exigência do exercício de habilidades e competências próprias aos $\mathrm{CP}$. A adoção da abordagem dos $\mathrm{CP}$ imprime às práticas de saúde mais qualidade, e também possibilita melhor continuidade do cuidado, e busca romper com o modelo biomédico, centrado na patologia e sua cura, em benefício a um modelo de atenção centrado no cuidado em saúde, na pessoa e, sobretudo, na experiência da doença, sentida por todos os envolvidos nesse processo ${ }^{3}$.

Além disso, segundo as novas Diretrizes Curriculares Nacionais $(\mathrm{DCN})^{13}$ para cursos de graduação em Educação Física, é preconizado que a formação deste profissional, em Instituições de Ensino Superior (IES) públicas e/ou privadas, seja pautada no arcabouço teórico e metodológico do SUS, e atualizada de acordo com as novas demandas que emergem e as perspectivas de mudança apresentadas pelas evoluções científicas e tecnológicas, o que reforça a importância de adequação das DCN às necessidades de saúde da população e aos objetivos e diretrizes do SUS.

Ao se analisar as novas Diretrizes Nacionais Curriculares propostas para o curso de Educação Física, em dezembro de $2018^{13}$, observa-se escassez de conteúdos voltados ao cuidado a doenças e/ou condições que colocam em risco a vida, em especial relacionados às suas fases de progressão e fim de vida. Inexistem referências à morte, ao processo de progressão de doenças e condições e terminalidade de vida, tampouco ao luto. Dessa forma, a formação do PEF pode apresentar limitações, o que poderá se refletir como fragilidades no trabalho em saúde posteriormente prestado por estes profissionais.

A atuação do PEF nos CP deve basear-se nas melhores práticas clínicas e abranger uma perspectiva de cuidado centrado na pessoa, suas necessidades e dimensões físicas, emocionais, sociais e espirituais. $\mathrm{Su}-$ gere-se a adoção de uma abordagem baseada no conhecimento da biografia do paciente e seus familiares/ cuidadores; aspectos biopsicossociais, espirituais e clínico-epidemiológico relacionados à doença e/ou tratamento; questões relacionadas a experiências prévias e nível motivacional para a prática de atividades físicas; realização de avaliações e testes funcionais, sendo desejável o compartilhamento dos resultados das avaliações e escuta acerca das expectativas, preferências e desejos dos pacientes e seus familiares; construção compartilhada - com paciente, familiares e equipe multiprofissional - do Projeto Terapêutico Singular (PTS); bem como reavaliações para ajustes e atualizações do PTS.

Dessa forma, faz-se necessário o reconhecimento de que os familiares, cuidadores e, também, a própria equipe multiprofissional devem ser foco de atenção pelo PEF durante todo o processo de apoio. O PEF deve compreender que todos os envolvidos no processo de cuidado podem sofrer de forma multidimensional durante o percurso da doença, tratamentos e mesmo após o óbito, no luto. É importante fortalecer uma compreensão de que o sofrimento pode - e deve - ser identificado, prevenido e paliado o mais precocemente possível, fato ainda não abordado na formação deste profissional, de acordo com as $\mathrm{DCN}^{13}$.

Além disso, ressaltamos a necessidade de fortalecer um sentimento de pertencimento, dos PEF à área da saúde, como categoria profissional indispensável em todos os níveis de atenção à saúde, não apenas no que tange ao componente da prevenção e promoção, mas também na recuperação, reabilitação e Cuidados Paliativos, questões pouco aprofundadas pela $\mathrm{DCN}^{13}$. Tais lacunas observadas na atual DCN podem criar barreiras de atuação profissional e de possibilidades terapêuticas disponíveis para a população.

As DCNs em Educação Física devem possibilitar que os currículos propostos possam construir o perfil acadêmico e profissional dos egressos, em termos de competências, habilidades, atitudes e conhecimentos, construídos a partir de perspectivas e abordagens contemporâneas de formação pertinente e compatível com referenciais nacionais e internacionais, tornando-os capazes de atuar com qualidade, eficiência e resolutividade nos diversos campos de atuação profissional, sendo desejável a inclusão de conteúdos, habilidades e competências relacionadas aos Cuidados Paliativos como forma de orientação às IES na construção de suas grades curriculares e, quando necessário, sua adequação.

Outrossim, a $\mathrm{DCN}^{13}$, ressalta que o aluno de Educação Física deve ter aquisição e desenvolvimento de conhecimentos, atitudes e habilidades profissionais que vão desde participar, assessorar, coordenar, liderar e gerenciar equipes multiprofissionais de discussão, definição, planejamento e operacionalização de políticas públicas e institucionais nos campos da saúde, bem como, diagnosticar os interesses, expectativas e necessidades das pessoas, com vistas a planejar, prescrever, orientar, assessorar, supervisionar, controlar e avaliar projetos e programas de atividades físicas.

Além disso, a formação do PEF deve contemplar temas como políticas e programas de saúde; atenção bási- 
ca, secundária e terciária em saúde, saúde coletiva, SUS, dimensões e implicações biológica, psicológica, sociológica, cultural e pedagógica da saúde; integração ensino, serviço e comunidade; gestão em saúde; objetivos, conteúdos, métodos e avaliação de projetos e programas de Educação Física na saúde, evidenciando a necessidade de incorporação do tema Cuidados Paliativos.

\section{Considerações Finais}

A prática profissional, no contexto dos $\mathrm{CP}$, mostra-se como importante e necessária área de aprendizado na formação do PEF, no que tange ao cuidado durante o percurso das doenças e/ou condições, cuidados de fim de vida, e luto.

Embora não haja uma orientação curricular explícita para inserção da temática dos Cuidados Paliativos nos cursos de Educação Física em nosso país, é possível identificar pontos de aproximação entre a atual DCN e competências relacionadas aos CP. Entretanto, haja vista a DCN ser orientadora das escolhas curriculares das IES brasileiras, enfatizamos a necessidade de uma comunicação inequívoca e específica sobre a importância de inclusão dos $\mathrm{CP}$ como componente curricular dos cursos de Educação Física.

Dessa forma, para avançar na implementação dos $\mathrm{CP}$ no rol das competências do PEF, é importante que o Sistema CONFEF/CREFs, as IES, sociedades científicas e a Gestão do SUS, de forma compartilhada, advoguem pela introdução de conteúdos e competências relacionadas aos CP nos currículos das IES de Educação Física, favorecendo o desenvolvimento de ações voltadas ao ensino, pesquisa e extensão em CP na formação básica e continuada do referido profissional.

Além disso, sugerimos a realização de estudos e o desenvolvimento de orientações técnicas voltadas à atuação do PEF nos CP, no que tange a métodos, técnicas e possibilidades de intervenção, potencializando e ampliando as expectativas, experiências e conhecimento profissional.

\section{Conflito de interesse}

Os autores declaram não haver conflito de interesse.

\section{Contribuição dos autores}

Souza Filho BAB, foi responsável pela concepção do manuscrito e os demais autores participaram efetivamente da análise, interpretação dos dados, redação do manuscrito, revisão crítica do conteúdo e aprovação final da versão a ser publicada.

\section{Referências}

1. Gómez-Batiste X, Connor S. Building integrated palliative care programs and services. Worldwide Hospice Palliative Care Alliance; 2017.

2. WHO. Integrating palliative care and symptom relief into the response to humanitarian emergencies and crises: a WHO Guide. Geneva: World Health Organization; 2018.

3. Tiberini R, Richardson H. Rehabilitative Palliative Care: A Challenge for the 21st Century. Hospice UK; 2015.

4. Conselho Federal de Educação Física. Resolução no 391, de 26 de agosto de 2020. Seç. 12020 p. 400.

5. Boulay SD, Rankin M. Cicely Saunders: the founder of the modern Hospice Movement. United Kingdom: Hodder \& Stoughton; 2007.

6. Davies E, Higginson IJ. The Solid Facts: Palliative care. Copenhagen: World Health Organization; 2004.32 p.

7. WHO. Cancer pain relief and palliative care. Geneva: World Health Organization; 1990.

8. WHO. Cancer pain refief and palliative care report. Geneva: World Health Organization; 2002.

9. Cherny NI, Fallon M, Kaasa S, Portenoy RK, Currow D, organizadores. Oxford textbook of Palliative Medicine. Fifth edition. Oxford: Oxford University Press; 2015. 1254 p.

10. Franklin DJ, Cheville AL. Medical Rehabilitation and the Palliative Care Patient. In: Oxford Textbook of Palliative Medicine. 5o ed Oxford University Press; 2015. p. 1-31.

11. dos Santos AFJ, Ferreira EAL, Guirro ÚB do P. Atlas dos Cuidados Paliativos no Brasil 2019. Academia Nacional de Cuidados Paliativos; 2020.

12. Comissão Intergestores Tripartite. Resolução no 41, de 31 de outubro de 2018. 2018 p. 1.

13. BRASIL. Resolução no 6, de 18 dezembro de 2018. Seç. 1 2018 p. 48-9.

14. Clark D, Baur N, Clelland D, Garralda E, López-Fidalgo J, Connor S, et al. Mapping levels of palliative care development in 198 countries: the situation in 2017. Journal of Pain and Symptom Management. 2020;59(4):794-807.

15. Gamondi C, Larkin P, Paine S, European Association for Palliative Care. Core competencies in palliative care: an EAPC White Paper on palliative care education. European Journal of Palliative Care. 2013;20(2):86-145. 